\title{
The research on the active vibration isolator
}

\section{BaiWei Guo ${ }^{1, a}$, YongLiang Zhang ${ }^{1, b}$ and XiuYun Meng ${ }^{1, c}$}

(1. Key Laboratory of Dynamics and Control of Flight Vehicle, Ministry of Education

Beijing Institute of Technology, Beijing 100081, China)

aguobw@bit.edu.cn, b20901061@bit.edu.cn, cMengxy@bit.edu.cn

Keywords: Bi-layer structure, PID, vibration isolation, cross-coupling, synchronization

\begin{abstract}
The effect on the high-precision imaging load because of the vibrations from the satellite platforms is becoming more and more non negligible. In the paper, the bi-layer structure mixed with passive part and active part is adopted for the leg and the location of the actuator is studied. As for the control algorithm, the loop-shaping approach is introduced. Additionally, the cross-coupling control strategy is adopted to improve the vibration isolation performance for the vibration isolator with two legs.
\end{abstract}

\section{Introduction}

The requirements for the image quality is becoming higher and higher with the development of space imaging technology, so the effect on the high-precision imaging load because of the vibrations from the satellite platforms is becoming more and more non negligible. The way mixed with sensor, controller, and actuator is adopted to build the leg with the in-depth study of the active components, but the bandwidth of the isolator that has only active part is limited and the passive part, such as dampers or spring, is needed to deal with the high-frequency disturbances. So the attentions are paid on the way mixed with passive part and active part.

Many different control strategies were proposed, such as the blended velocity feedback [1], the fusion with the force signal and the acceleration signal [2], the PID control, state feedback control and the $\mathrm{H} \infty$ control etc [3] to improve the vibration isolation performance of the isolator and the advantages and disadvantages of different control strategies were discussed [4]. On the basis of the above, the dual closed-loop controller with the modified PID is introduced for the optimal bi-layer structure. For the isolator with many legs, the controller is complex and difficult. The cross-coupling synchronization algorithms were adopted for the multi-axis motion synchronization [5,6], but the algorithm is introduced to improve performance of the isolator with two legs by coordinating the two forces from the two legs. The article is organized as follows: in part 1 , the loop-shaping approach is introduced for the bi-layer structure; in part 2, the controller that is designed by the cross-coupling synchronization algorithm is introduced for the vibration isolator with two legs.

\section{The design of the loop-shaping approach for the bi-layer vibration isolation}

\section{Problem description}

In the paper, the disturbances from the base platform are considered and the disturbances from the payload are ignored. The structure of the vibration isolator is shown as Fig.1

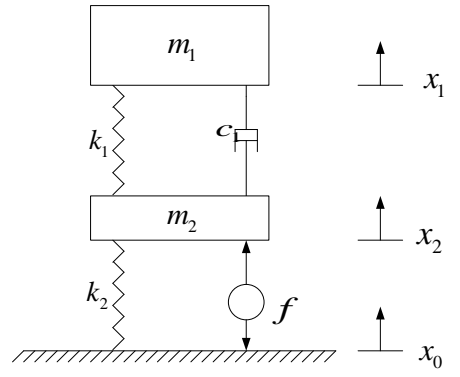

Fig.1. the structure of the vibration isolator 
and the dynamic equation can be got by Newton's law:

$$
\begin{gathered}
m_{1} \ddot{x}_{1}+c_{1}\left(\dot{x}_{1}-\dot{x}_{2}\right)+k_{1}\left(x_{1}-x_{2}\right)=0 \\
m_{2} \ddot{x}_{2}+k_{2}\left(x_{2}-x_{0}\right)-c_{1}\left(\dot{x}_{1}-\dot{x}_{2}\right)-k_{1}\left(x_{1}-x_{2}\right)=f_{a}
\end{gathered}
$$

Then G0(s), G1(s) and G2(s) can be expressed as Eq.3, Eq. 4 and Eq.5.

$$
\begin{aligned}
G_{0}(s) & =\frac{k_{2}\left(m_{1} s^{2}+c_{1} s+k_{1}\right)}{m_{1} m_{2} s^{4}+\left(m_{1} c_{1}+m_{2} c_{1}\right) s^{3}+\left(m_{1} k_{2}+m_{1} k_{1}+m_{2} k_{1}\right) s^{2}+k_{2} c_{1} s+k_{1} k_{2}} \\
G_{1}(s) & =\frac{m_{1} s^{2}+c_{1} s+k_{1}}{m_{1} m_{2} s^{4}+\left(m_{1} c_{1}+m_{2} c_{1}\right) s^{3}+\left(m_{1} k_{2}+m_{1} k_{1}+m_{2} k_{1}\right) s^{2}+k_{2} c_{1} s+k_{1} k_{2}} \\
G_{2}(s) & =\frac{c_{1} s+k_{1}}{m_{1} s^{2}+c_{1} s+k_{1}}
\end{aligned}
$$

Where $G_{0}(s)$ is the transfer function from the disturbance to the displacement $x_{2}$ without the actuator, $G_{1}(s)$ is the transfer function from the output of the actuator to the displacement $x_{2} ; G_{2}(s)$ is the transfer function from the displacement $\mathrm{x}_{2}$ to the displacement $\mathrm{x}_{1}$ (measured by the sensor).

\section{Design of the controller}

The output of the actuator fa depends on the signals from the displacement sensors. The block diagram of the system is shown as Fig.4.

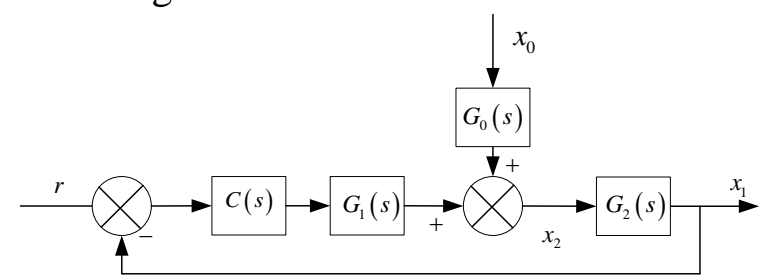

Fig.2. the block diagram of the system

In Fig.2, $\mathrm{x} 0$ is the disturbance signal; $\mathrm{C}(\mathrm{s})$ is the controller to be designed and the parameters of the leg are shown in table 1

Table.1. the parameters of the leg

\begin{tabular}{cccccc}
\hline $\mathrm{m}_{1}(\mathrm{~kg})$ & $\mathrm{m}_{2}(\mathrm{~kg})$ & $\mathrm{c}_{1}(\mathrm{~N} . \mathrm{s} / \mathrm{m})$ & $\mathrm{c}_{2}(\mathrm{~N} . \mathrm{s} / \mathrm{m})$ & $\mathrm{k}_{1}(\mathrm{~N} / \mathrm{m})$ & $\mathrm{k}_{2}(\mathrm{~N} / \mathrm{m})$ \\
\hline 1 & 0.1 & 2 & 0 & 100 & 1000 \\
\hline
\end{tabular}

The design process of the controller which is used to isolate the disturbance is divided into two steps

Step.1. original design: as a excellent system, the magnitude-frequency characteristics of the open loop is much the same with the magnitude-frequency characteristics of the transfer function Gd(s) when the frequency is smaller than the cross-over frequency, then the Eq.6 is gotten as follows

$$
|L(s)|=\left|G_{d}(s)\right|
$$

where $\mathrm{L}(\mathrm{s})=\mathrm{C}(\mathrm{s}) \mathrm{G}_{1}(\mathrm{~s}) \mathrm{G}_{2}(\mathrm{~s})$ and $\mathrm{G}_{\mathrm{d}}(\mathrm{s})=\mathrm{G}_{0}(\mathrm{~s}) \mathrm{G}_{2}(\mathrm{~s})$. So we can get $\mathrm{C}_{0}(\mathrm{~s})=\mathrm{G}_{0}(\mathrm{~s}) \mathrm{G}_{1}{ }^{-1}(\mathrm{~s})$.

Step.2. Enlarging the gain in the low-frequency stage. The transfer function $(\mathrm{s}+\mathrm{w} 0) / \mathrm{s}$ is added on the transfer function $\mathrm{CO}(\mathrm{s})$ in order to gain the integral action. Although a large w0 benefits the system in the low-frequency stage, it is destructive to the phase stability of the system. So an appropriate value for w0 is $0.2 \mathrm{wc}$, where wc is the expected shear frequency. As for the system in the paper, the shear frequency wc $=5$ is selected. So the revised controller is $C(s)=(s+1 / s) * C 0(s)$.

The amplitude-frequency characteristics of the open loop and the closed loop are shown in the Fig.3. The closed-loop transfer function from $\mathrm{x} 0$ to $\mathrm{x} 1$ is $\mathrm{S}(\mathrm{s})$, and the open-loop transfer function from $\mathrm{x} 0$ to $\mathrm{x} 1$ is $\mathrm{S} 0(\mathrm{~s})$. 


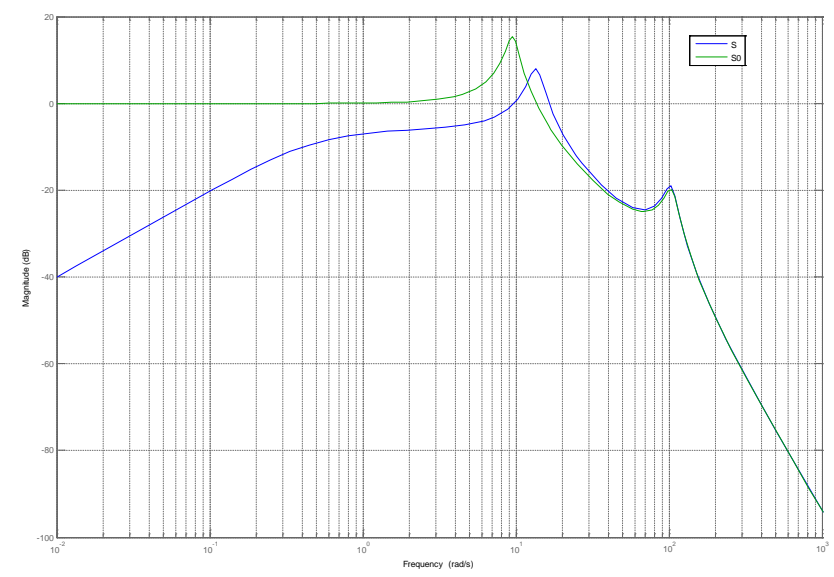

Fig.3. the amplitude-frequency characteristics of the transfer function $\mathrm{S}(\mathrm{s})$ and $\mathrm{S}_{0}(\mathrm{~s})$

From the Fig.3, it is concluded that the performance in vibration isolating is improved to a great extent in the low-frequency stage.

\section{The cross-coupling synchronization controller for the isolator with two legs}

The design of the controller for single leg is introduced and the performance in vibration isolation is perfect, but the controller will become complicated if the isolator has more than one leg. As Fig. 4 shows, the isolator with two legs is designed to control the vibration in one rotation direction.

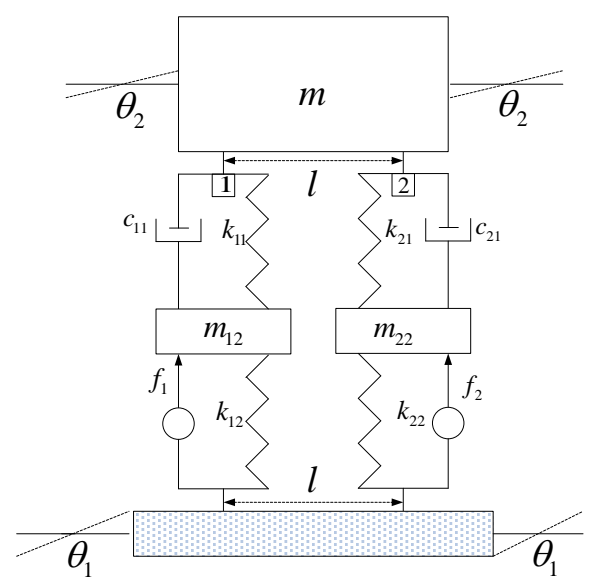

Fig.4. the isolator with two legs

Then the state equation of No 1 leg is built as follows

$$
\begin{aligned}
& \dot{X}_{1}=A_{1} X+B_{1} u+E_{1} X_{10} \quad F_{1}=C_{1} X_{1} \\
& X_{1}=\left[\begin{array}{c}
x_{11} \\
\dot{x}_{11} \\
X_{12} \\
\dot{x}_{12}
\end{array}\right], A_{1}=\left[\begin{array}{cccc}
0 & 1 & 0 & 0 \\
\frac{k_{11}}{m_{11}} & \frac{c_{11}}{m_{11}} & -\frac{k_{11}}{m_{11}} & -\frac{c_{11}}{m_{11}} \\
0 & 0 & 0 & 1 \\
-\frac{k_{11}}{m_{12}} & -\frac{c_{11}}{m_{12}} & \frac{k_{11}+k_{12}}{m_{12}} & \frac{c_{11}}{m_{12}}
\end{array}\right], B_{1}=\left[\begin{array}{c}
0 \\
0 \\
0 \\
1 \\
\frac{m_{12}}{2}
\end{array}\right], E_{1}=\left[\begin{array}{c}
0 \\
0 \\
0 \\
\frac{k_{12}}{m_{12}}
\end{array}\right], C_{1}=\left[\begin{array}{c}
-k_{11} \\
-c_{11} \\
k_{11} \\
c_{11}
\end{array}\right]^{T}
\end{aligned}
$$

For No 2 leg, the corresponding state equation is similar to the No 1leg

The actual values of the parameters in the legs may differ from the design values, so we assume that the actual values of the parameters in No 1 leg are equal to the design values and the actual values of the parameters in No 2 leg increase by $10 \%$ basing on the design values. Then the parameters of the two legs are shown in table 2

Table 2. parameters of the two legs 


\begin{tabular}{lccccc}
\hline NO & $\mathrm{m}_{1}(\mathrm{~kg})$ & $\mathrm{m}_{2}(\mathrm{~kg})$ & $\mathrm{k}_{1}(\mathrm{~N} / \mathrm{m})$ & $\mathrm{k}_{2}(\mathrm{~N} / \mathrm{m})$ & $\mathrm{c}_{1}(\mathrm{~N} . \mathrm{s} / \mathrm{m})$ \\
\hline 1 & 1.0 & 0.1 & 100 & 1000 & 2 \\
2 & 1.1 & 0.11 & 110 & 1100 & 2.2
\end{tabular}

Introducing the PID cross-coupling synchronization control algorithm

$$
\begin{aligned}
& u_{1}(k)=u_{1}(k-1)+k_{p}\left[e_{1}(k)-e_{1}(k-1)\right]+k_{i} e_{1}(k)+\frac{k_{d}\left(e_{1}(k)-2 e_{1}(k-1)+e_{1}(k-2)\right)}{T_{s}}+k_{c}\left(F_{1}(k)-F_{2}(k)\right) \\
& u_{2}(k)=u_{2}(k-1)+k_{p}\left[e_{2}(k)-e_{2}(k-1)\right]+k_{i} e_{2}(k)+\frac{k_{d}\left(e_{2}(k)-2 e_{2}(k-1)+e_{2}(k-2)\right)}{T_{s}}+k_{c}\left(F_{2}(k)-F_{1}(k)\right)
\end{aligned}
$$

$k_{p}$ is the coefficient of the proportional part, $k_{i}$ is the coefficient of the integral part, and $k_{c}$ is the synchronous coupling coefficient . The value of the synchronous coupling coefficient has a great influence on the output of the actuators and the performance on vibration isolation. Then taking the mean square error of the output of the actuators and the mean square error of the angular displacement of the payload platform as the evaluation criteria. We do some simulation test for different $\mathrm{k}_{\mathrm{c}}$ and the result is shown in the table 3 .

Table 3. the mean square errors of the output force of the actuator and the angular displacement of the payload for different $\mathrm{k}_{\mathrm{c}}$

\begin{tabular}{lllccll}
\hline $\mathrm{k}_{\mathrm{c}}$ & -5 & -10 & -20 & -30 & -50 & -60 \\
\hline$\left|\operatorname{std}\left(\mathrm{u}_{1}\right)-\operatorname{std}\left(\mathrm{u}_{2}\right)\right|$ & 8.19 & 8.83 & 11.60 & 16.28 & 48.38 & $\infty$ \\
$\operatorname{std}\left(\mathrm{u}_{1}\right)$ & 52.80 & 62.62 & 84.13 & 112.26 & 285.65 & $\infty$ \\
$\operatorname{std}\left(\mathrm{u}_{2}\right)$ & 61.0 & 71.46 & 95.63 & 128.50 & 334.41 & $\infty$ \\
$\operatorname{std}\left(\theta_{2}\right) \times 10^{5}$ & 3.35 & 1.55 & 1.10 & 0.63 & 0.37 & $\infty$ \\
\hline
\end{tabular}

(Note:the symbol std stand for the mean square error)

From the result in the table 3, we can see that the greater the value of coupling coefficient is, the better the performance is, but the greater the outputs of the actuator are. In addition, the system will become unstable if the value of coupling coefficient becomes greater than a value. Considering the capability of the actuator and the vibration isolation performance,we set $\mathrm{kc}=-30$ in the paper . The parameters of the the cross-coupling synchronization controller are $\mathrm{kp}=1000, \quad \mathrm{ki}=1000 \mathrm{and} \mathrm{kc}=-30$.

\section{Conclusions}

In the paper, the position of the actuator for the bi-layer structure is studied and a optimal structure is proposed. For the optimal structure, the modified PI controller is designed by the frequency domain method. There is $20 \mathrm{~dB}$ attenuation for the white noise disturbance with the modified PI controller. For the isolator with two legs, we design the PI cross-coupling synchronization controller and study the effect on the performance and output forces of the actuators because of the coefficient of cross-coupling.

\section{Acknowledgements}

This work was financially supported by the Beijing Institute of Technology academic research fund (item number: 20130142015).

\section{References}

[1] Alujevic, N., et al., Stability and performance limits for active vibration isolation using blended velocity feedback. Journal of Sound and Vibration, 2011. 330(21): p. 4981-4997.

[2] Tjepkema, D., J. van Dijk, and H.M.J.R. Soemers, Sensor fusion for active vibration isolation in precision equipment. Journal of Sound and Vibration, 2012. 331(4): p. 735-749.

[3] Kerber, F., et al., Control concepts for an active vibration isolation system. Mechanical Systems and Signal Processing, 2007. 21(8): p. 3042-3059.

[4] Preumont, A., et al., Force feedback versus acceleration feedback in active vibration isolation. 
Journal of Sound and Vibration, 2002. 257(4): p. 605-613.

[5] Barton, K.L. and A.G. Alleyne, A Cross-Coupled Iterative Learning Control Design for Precision Motion Control. Ieee Transactions on Control Systems Technology, 2008. 16(6): p. 1218-1231.

[6] Xiao, Y., K.Y. Zhu, and H.C. Liaw, Generalized synchronization control of multi-axis motion systems. Control Engineering Practice, 2005. 13(7): p. 809-819. 